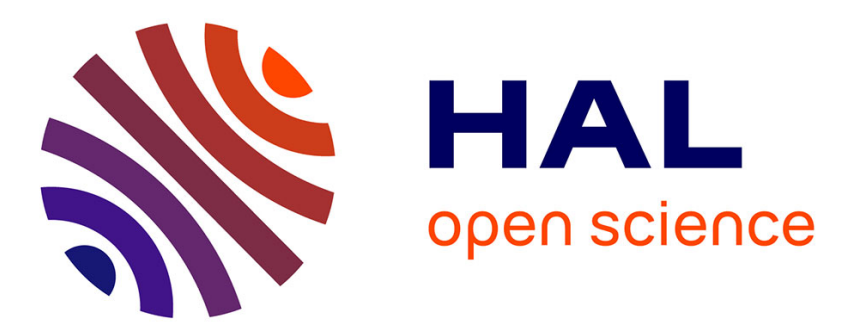

\title{
AN ASYMPTOTIC ANALYSIS FOR ONE DIMENSIONAL STRESS WAVE PROPAGATION IN DAMAGED VISCOELASTIC MEDIA
}

\author{
M. Lu, Z. Duan
}

\section{> To cite this version:}

M. Lu, Z. Duan. AN ASYMPTOTIC ANALYSIS FOR ONE DIMENSIONAL STRESS WAVE PROPAGATION IN DAMAGED VISCOELASTIC MEDIA. Journal de Physique IV Proceedings, 1991, 01 (C3), pp.C3-861-C3-866. 10.1051/jp4:19913120 . jpa-00249925

HAL Id: jpa-00249925

https://hal.science/jpa-00249925

Submitted on 1 Jan 1991

HAL is a multi-disciplinary open access archive for the deposit and dissemination of scientific research documents, whether they are published or not. The documents may come from teaching and research institutions in France or abroad, or from public or private research centers.
L'archive ouverte pluridisciplinaire HAL, est destinée au dépôt et à la diffusion de documents scientifiques de niveau recherche, publiés ou non, émanant des établissements d'enseignement et de recherche français ou étrangers, des laboratoires publics ou privés. 


\title{
AN ASYMPTOTIC ANALYSIS FOR ONE DIMENSIONAL STRESS WAVE PROPAGATION IN DAMAGED VISCOELASTIC MEDIA
}

\author{
M. LU* and Z.P. DUAN** \\ *Institute for Solids Mechanics, Dept Flight vehicle \\ Design \& Appl. Mech., Beijing Univ. of Aero. \& Astro., Beijing \\ 100083, China \\ ** LNM, Institute of Mechanics, CAS, Beijing, 100080, \\ China
}

\section{RESUME :}

Une technique de perturbation régulière est proposée pour traiter le problème de propagation d'une onde de contrainte uniaxiale dans un milieu viscoélastique avec endomnagement. Les caractéristiques de l'amortissement d'une onde sont étudiées à partir de l'obtention d'une solution asymptotique du premier ordre. De fait, il existe trois phénomènes dépendants du temps qui interviennent dans la réponse dynamique des matériaux, le premier exprime les caractéristiques de la propagation de l'onde, le second traduit l'effet naturel de la matrice viscoélastique et le troisième vient de la dépendance de l'endommagement par rapport au temps. La comparaison d'une solution asymptotique du premier ordre avec les résultats numériques obtenus par un calcul en différences finies montre que la technique "de dilatation perturbatrice" peut offrir une approche pratique du problème posé.

\begin{abstract}
A regular perturbation technique is suggested to deal with the problem of one dimensional stress wave propagation in viscoelastic media with damage. Based upon the first order asymptotic solution obtained, the characteristics of wave attenuation are studied. In fact, there exist three different time-dependent phenomena featuring the dynamic response of the materials, the first expressing the characteristics of wave propagation, the second indicating the innate effect of visco-elastic matrix and the third coming from the time dependent damage. The comparision of first order asymptotic solution with the numerical results calculated by a finite difference procedure shows that the perturbation expansion technique may offer a useful approach to the problem concerned.
\end{abstract}

\section{Introduction}

The study on stress wave propagation in viscoelastic media with damage is increasingly becoming an important research subject with practical interests. For example, the failure analysis of highly filled polymers such as rocket propellents, the study of blastic wave propagation in rock-liked media as well as the characterization of dynamic response of porous materials such as tungsten alloy subjected to impact loading typically show some applications of this investigation.

Unlike the linear viscoelastic media without damage, a strong nonlinearity and a different type of history dependence are involved in the problem due to introduction of damage and its nonlinear coupling effects with viscoelasticity of matrix. A little progress has so far been known towards getting a better understanding of the main features of stress wave propagation in such a media partly because of the lack of knowledge of an accurate description of physical modelling concerning with damage and its evolution law and partly because of the complexity and difficulty resulted from the nonlinearity of the problem as just stressed.

Previous studies on the problem were conducted using a pure numerical procedure usually provided by finite difference method ${ }^{[6]}$, which is not always effective and convenient. To overcome this kind of shortage occured in pure numerical procedures it seems necessary to develop an alternative way to deal with this problem.

For simplicity the present study will focus on one-dimensional stress wave propagation and suggests a regular 
perturbation technique taking a damage rate controlling factor as a small perturbation parameter when the damage keeps small in comparison with a unity. After expanding the governing equations and the associated initial and boundary conditions in terms of a chosen small parameter, a series of asymptotic equations with various order approximations can be obtained and solved consequently, the attenuation of stress at wave front and other features by means of Laplace transformation technique as well as damage evolution can be wellillustrated. Hopefully the perturbation expansion method and the obtained results will be helpfull to get a better understanding of main features of wave attenuation in such a media.

\section{Governing Equations}

Based on continuum damage mechanics (see Kachanov [1] and Chaboche ${ }^{[2]}$ ), the basic equation for onedimensional stress wave propagation in damaged-viscoelastic media may be simplified to the following forms

$$
\begin{array}{ll}
\text { Momentum: } & \frac{\partial v}{\partial t}=\frac{1}{\rho_{o}} \frac{\partial \sigma}{\partial x} \\
\text { Continuity: } & \frac{\partial \epsilon}{\partial t}=\frac{\partial v}{\partial x} \\
\text { Constitutive law: } & \sigma=(1-D) \int_{o}^{t} E^{t}(\tau) \frac{d \epsilon}{d \tau} d \tau \\
\text { Damage evolution: } & \frac{\partial D}{\partial t}=f(\sigma, D)=\left(\frac{\sigma}{A}\right)^{u}(1-D)^{-\alpha}
\end{array}
$$

where $t$ is the time, $x$ is the Lagrangian coordinate taken in the direction of wave propagation, $\rho_{o}$ is the initial mass density. Obviously, there are four time-position dependent variables to be determined, that is ,the stress $\sigma$, the particle velocity $v$, total strain $\epsilon$ as well as the damage quantity $D$, where $D$ is usually as void ratio per unit volume. In equation (3), $E^{t}(\tau) \cong E(t-\tau), 0 \leq \tau<\infty$ is the relaxation spectrum function of visco-elastic matrix, and $\alpha, \mu, A$ in equation(4) are the material coefficients in relation with creep type evolution of damage as suggested by Kachanov [3], et al. From equation (1)-(4), it is readily seen that the stress has chosen to depend both on the matrix relaxation function and the damage measure, so that nonlinearity of (1) (4) is arisen from the coupling effect of viscoelasticity of matrix with damage. For simplicity, we limited in the following discussion ourselves the case where the stress wave propagates along a semi-infinite bar, then the initial and velocity boundary conditions are correspondingly given below

$$
\begin{array}{cccc}
x=0, & v=v_{o} g(t) & \text { or } \quad & \sigma=\sigma_{0} h(t) \quad \text { and } \sigma, \quad v \rightarrow 0 \text { as } x \rightarrow \infty \\
t=0, & x>0, & \sigma=v=0, & D=D_{0}=\mathrm{Const}
\end{array}
$$

where $D_{0}$ is the initial damage measure, $v_{0}$ is the amplitude of particle velocity imposed at one end of the bar. The basic equation (1) (4) and I.B. conditions (5) can be nondimensionalized by introducing a set of new independent and dependent variables

$$
\bar{x}=\frac{x}{C_{o} \tau}, \quad \bar{t}=\frac{t}{\tau}, \quad \bar{\sigma}=\frac{\sigma}{\rho_{o} c_{o} v_{o}}, \quad \bar{v}=\frac{N}{v_{o}}, \quad \bar{\epsilon}=\frac{c_{o}}{v_{o}} \epsilon, \quad \bar{D}=\frac{D-D_{o}}{1-D_{o}}, \quad \bar{E}^{t}=\frac{E^{t}\left(1-D_{o}\right)}{\rho_{o} c_{o}^{2}}
$$

where $\tau$ represents a characteristic relaxation time of viscoelastic matrix, which is prescribed in the relaxation spectrum function $E^{t}(\tau)$, and $c_{o}=\left[E_{0} / \rho_{0}\left(1-D_{0}\right)\right]^{\frac{1}{2}}$ is the leading wave speed. Thus, using (6) the basic equations (1) (4) and I.B. Conditions (5) could be nondimensionalized and reduce to the following forms

$$
\begin{aligned}
& \frac{\partial \bar{v}}{\partial \bar{t}}=\frac{\partial \bar{\sigma}}{\partial \bar{x}}, \quad \frac{\partial \bar{\epsilon}}{\partial \bar{t}}=\frac{\partial \bar{v}}{\partial \bar{x}} \\
& \bar{\sigma}=(1-\bar{D}) \int_{0}^{\bar{t}} \bar{E} \frac{\partial \bar{\epsilon}}{\partial \bar{\tau}} d \tau \\
& \bar{D}=\delta \bar{\sigma}^{\mu}(1-\bar{D})^{-\alpha}
\end{aligned}
$$

and

$$
\begin{aligned}
& \bar{x}=0, \quad \bar{v}=\bar{g}(\bar{t}), \quad \text { or } \quad \bar{\sigma}=\bar{h}(\bar{t}) \\
& \bar{x} \rightarrow \infty, \quad \bar{v} \rightarrow 0, \\
& \bar{t}=0, \quad \bar{\sigma}=\bar{v}=\bar{D}=\bar{\epsilon}=0 \quad \text { for } \quad \bar{x}>0
\end{aligned}
$$

In equation(17), $\delta=\frac{\tau}{\left(1-D_{0}\right)^{\alpha}}\left[\frac{\rho_{0} c_{0} v_{0}}{A}\right\}^{\mu}$ is a nondimensional parameter that can be used as a perturbation parameter, we may call it the damage rate controlling factor. 


\section{Perturbation Expansion}

In this section, a regular perturbation expansion is developed to solve equation(7) with I.B. Conditions(8). First, it can be shown from equation(7) that value of $\delta$ is of the same order as the nondimensional damage quantity $D$ that is supposed to be small in comparison with a unity. Therefor, $\delta$ can be taken as a small perturbation parameter, with which all solutions for the problem(7) and (8) can be expanded by

$$
[\sigma, v, \epsilon, D]=\sum_{n=0}^{\infty}\left[\sigma_{n}, v_{n}, \epsilon_{n}, D_{n}\right] \delta^{n}
$$

It should be pointed out that all bars marked over all variables in (6) (8) have been moved out in (9) as well as in the later calculation for the sake of convenience and this should not lead any confusion for a further analysis.

Integrating equation (7) one obtains an analytical expression for damage $D$ in terms of the history of stress

$$
D=1-\left[1-(1+\alpha) \delta \int_{0}^{t} \sigma^{\mu}(\tau, x) d \tau\right]^{\frac{1}{1+\alpha}}
$$

If substituting the perturbation expansion (9) into equation (10) and comparing the coefficients of $\delta^{n}$ $(\mathrm{n}=0,1,2, \ldots)$ on both sides of this equation, one can show that $D_{n}$ only depends on the histories of $\sigma_{0}, \sigma_{1} \ldots, \sigma_{n-1}$ and has nothing to do with $\sigma_{n}$. For example, taking $n=1$ and $n=2$ we have

$$
\frac{\partial D_{1}}{\partial t}=\sigma_{o}^{\mu}, \quad \frac{\partial D_{2}}{\partial t}=\sigma_{o}^{\mu-1} \sigma_{1}-2 \alpha \sigma_{o}^{\mu} D_{1}
$$

or

$$
D_{1}=\int_{0}^{t} \sigma_{o}^{\mu}(x, \tau) d \tau, \quad D_{2}=\int_{0}^{t} \sigma_{o}^{\mu-1}(x, \tau) \sigma_{1}(x, \tau) d \tau-2 \alpha \int_{0}^{t} \sigma_{0}^{\mu}(x, \tau) D_{1}(x, \tau) d \tau
$$

which means that $D_{1}$ only depends on the history of $\sigma_{0}$, and $D_{2}$ on the histories of $\sigma_{0}$ and $\sigma_{1}$ only. Imposing the regular procedure of perturbation techniques on equation(7), one can obtain a series of recurrence equations

$$
\begin{aligned}
& \frac{\partial v_{n}}{\partial t}=\frac{\partial \sigma_{n}}{\partial x}, \quad \frac{\partial \epsilon_{n}}{\partial t}=\frac{\partial v_{n}}{\partial x_{n}} \\
& \sigma_{n}=\int_{0}^{t} E^{t}(\tau) \frac{\partial v_{n}}{\partial x} d \tau-\sum_{\substack{m+h=n \\
m \neq 0}} D_{m} \int_{0}^{t} E^{t}(\tau) \frac{\partial v_{k}}{\partial x} d \tau
\end{aligned}
$$

for the n-th order approximations $\left(v_{n}, \sigma_{n}, \epsilon_{n}\right)$. Obviously, these equations can be reduced to a single linear integro-differential equation for $v_{n}$ or $\sigma_{n}$,

$$
\mathcal{F}\left(v_{n}\right) \Longleftrightarrow \frac{\partial v_{n}}{\partial t}-\int_{0}^{t} E^{t}(\tau) \frac{\partial^{2} v_{n}}{\partial x^{2}} d \tau=\mathcal{L}_{n}^{(v)}\left(v_{o}, v_{1}, \ldots v_{n-1}\right)
$$

and

$$
\mathcal{F}\left(\sigma_{n}\right) \Longleftrightarrow \frac{\partial \sigma_{n}}{\partial t}-\int_{0}^{t} E^{t}(\tau) \frac{\partial^{2} \sigma_{n}}{\partial x^{2}} d \tau=\mathcal{L}_{n}^{(\sigma)}\left(\sigma_{o}, \sigma_{1}, \ldots \sigma_{n-1}\right)
$$

where the integro-differential operator $F$ is linear with respect to $v_{n}$ or $\sigma_{n}$ and the nonhomogenous terms $\mathcal{L}_{(n)}^{(v)}$ on the right hand side of equations $(14) \sim(15)$ are determined only through the zero-th to $(\mathrm{n}-1)$-th order approximation solutions

$$
\begin{array}{ll}
\mathcal{L}_{n}^{(v)}=-\frac{\partial}{\partial x}\left[\sum_{m+k=n} D_{m} \int_{0}^{t} E^{t}(\tau) \frac{\partial v_{k}}{\partial x} d \tau\right] & n \neq 0 \\
\mathcal{L}_{n}^{(\sigma)}=-\frac{\partial}{\partial t} \sum_{m+k=n} D_{m} \int_{0}^{t} E^{t}(\tau) \frac{\partial v_{k}}{\partial \tau} d \tau & n \neq 0
\end{array}
$$

The I.B. conditions for $v_{n}$ or $\sigma_{n}$ can also be determined correspondingly through equation (8)

$$
\left.V_{n}\right|_{x=0}=\left\{\begin{array}{ll}
g(t) & n=0 \\
0 & n>0
\end{array} \quad \text { or }\left.\quad \sigma_{n}\right|_{x=0}= \begin{cases}h(t) & n=0 \\
0 & n>0\end{cases}\right.
$$

and

$$
v_{n}=\sigma_{n}=\epsilon_{n}=0 \quad \text { when } \quad t=0
$$


Since equation (13) (14) with conditions (17) (18) are linear for $v_{n}$ or $\sigma_{n}$, they can be, in principle, solved using Laplace transformation method without any essential difficulties. To show this, when either the velocity boundary condition or stress boundary condition is prescribed. By solving the homogeneous eqnations $(14)(\mathrm{n}=0)$ with I.B.conditions(18), we may easily write down the zero-th order approximation solutions

or

$$
v_{o}(x, t)=L^{-1} \tilde{g}(s) e^{-\frac{s}{\sqrt{\hat{E}(s)}} x}
$$

$$
\sigma_{o}(x, t)=L^{-1} \tilde{h}(s) e^{-\frac{s}{\sqrt{\hat{E}(s)}} x}
$$

where $\hat{E}(s)$ is the Laplace transformation of $E(t)$ and $L^{-1}$ represents Laplace inverse transformation operator, and $\tilde{g}(s)$ and $\tilde{h}(s)$ corresponds, respectively, the Laplace transformation of $g(t)$ and $h(t)$. Using (20) or (21) , the various order approximation solutions can be derived through an iteration approach to equations (14) $\sim(19)$.

\section{Example and Discussion}

An example will be given below illustrating the application of the perturbation procedure described above. To do this, it is necessary first to give a concrete form of relaxation modulus $E^{t}=E(t-\tau)$. For some viscoelastic materials, they are usually specified to the form

$$
E(t)=\sum_{i=1}^{m} E_{i} e^{-\frac{t}{r_{i}}}
$$

where $\tau_{i}$ and $E_{i}(i=1,2, \ldots)$ are known as the different relaxation times and the corresponding relaxation modulus. For Maxwell-type materials, equation(22) takes only one term

$$
E(t)=E_{o} e^{-\frac{t}{t}}
$$

in which $\tau$ is a typical relaxation time that has been used in equation(6) as a characteristic time for nondimensionalization .Therefore, the nondimensional form of equation(23) is given as follow

$$
\bar{E}(t)=E e^{-t}
$$

obviously, the Laplace transformation of function $E(t)$ given in $(22)$ is

$$
\hat{E}(s)=\sum_{i} \frac{E_{i} r_{i}}{1+s \tau_{i}}
$$

On the other hand, the nondimensional velocity boundary condition imposed at $x=0$ is assumed to be

$$
\left.v\right|_{x=0}=g(t)=H(t)-H(t-T)
$$

where $H(t)$ is Haviside step function, and $T$ is the duration of the constant velocity imposed. The Laplace transformation of $g(t)$ is

$$
\tilde{g}(s)=\frac{1}{s}\left[1-e^{-T s}\right]
$$

Substitution of (25) and (27) into (20) leads to the zero-th order approximation in the form

$$
v_{o}(x, t)=\frac{1}{2 \pi_{i}} \int_{\varepsilon-i \infty}^{\varepsilon+i \infty} \frac{1}{s}\left(1-e^{\tau s}\right) e^{s t-a(s) x} d s, \quad \text { with } a(s)=\frac{s}{\sqrt{\Sigma \frac{E_{i} \tau_{i}}{1+s \tau_{i}}}}
$$

when the first term in (25) is kept only, the solution(28) for $\sigma_{0}$ and $v_{o}$ can be directly integrated as follows

$$
\begin{gathered}
\sigma_{o}(x, t)=\hat{I}_{o}(x, t)-\hat{I}_{o}(x, t-\tau) \\
v_{o}(x, t)=\int_{0}^{x}\left[\sqrt{E_{o}} \sigma_{o}(\xi, t)+\frac{1}{E_{o}} \frac{\partial \sigma_{o}(\xi, t)}{\partial t}\right] d \xi
\end{gathered}
$$


with

$$
\hat{I}_{0}(x, t)=E_{0}^{\frac{1}{2}} e^{-\frac{t}{3}} I_{0}\left(\frac{1}{2} \sqrt{t^{2}-x^{2}}\right) H(t-x)
$$

where $I_{o}$ is the modified zero-th order Bessel function of the first kind.

After obtaining the solutions (29)-(30) and using the described iteration procedure we can easily derive from equation (14)-(19) the first order approximation solutions, of which the first order approximations of damage $D_{1}$ stress $\sigma_{1}$ can be written in the forms

$$
\begin{aligned}
& D_{1}(x, t)=\int_{0}^{t}\left[\hat{I}_{o}(x, \tau)-\hat{I}_{o}(x, \tau-t)\right]^{\mu} d \tau \\
& \sigma_{1}(x, t)=E_{o} \frac{\partial}{\partial x}\left[v_{1}(x, t)-\int_{0}^{t} \exp (\tau-t) u_{1}(x, t) d \tau\right]-\sigma_{o}(x, t) D_{1}(x, t)
\end{aligned}
$$

where $u_{1}(x, t)$ is the first order approximation of displacement given by

$$
\begin{aligned}
u^{(1)}(x, t)= & \frac{1}{2} \int_{0}^{t}\left\{\int_{x}^{\infty} \hat{I}_{0}(\xi, t-\tau+\xi+x) f(\xi, \tau) H(t-\tau-\xi+x) d \xi\right. \\
& +\int_{0}^{x} \hat{I}_{0}(\xi, t-\tau+x+\xi) f(\xi, \tau) H(t-\tau-x+\xi) d \xi \\
& \left.-\int_{0}^{\infty} \hat{I}_{0}(\xi, t-\tau-x-\xi) f(\xi, \tau) H(t-\tau-x-\xi) d \xi\right\} d \tau
\end{aligned}
$$

Based on the solutions (29)-(30), we may see that

$$
\begin{aligned}
& \lim _{t \rightarrow \infty} D=8 \int_{0}^{\infty}\left[\hat{I}_{0}(x-\tau), \hat{I}_{0}(x, \tau-T)\right]^{\mu} d \tau+0\left(\delta^{2}\right) \neq 0 \\
& \lim _{t \rightarrow \infty} \sigma_{0}(x, t)=\lim _{t \rightarrow \infty} v_{0}(x, t)=0
\end{aligned}
$$

so that

$$
\lim _{t \rightarrow \infty} \sigma(x, t)=\delta \lim _{t \rightarrow \infty} \sigma_{1}(x, t)+o\left(\delta^{2}\right)
$$

Therefore, the viscoelastic effects of matrix on the behaviors of solutions will gradually vanish with the increase of time, this is due to its characteristics of fading memory. However, the permanent damage left inside material will cause a residual stress distribution. This is an important difference between the characteristics of wave propagation in undamaged viscoelastic materials and in damaged materials. In order to test what an accuracy in the first-order approximation solution given in (33)-(35), a numerical computation has been also conducted for the same problem using a finite difference method. The comparisons of the first order asymptotic solutions with the results from pure finite difference numerical analysis are depicted in Fig.1-Fig.4 of Appendix, assuming $\mu=2.0, \alpha=1.2, E_{1}=1.0 \times 10^{9} \mathrm{~N} / \mathrm{m}^{2}, T=1$ and $\delta=0.2$. Fig.1-Fig.3 show the comparisons of the changes of strain, stress and damage with time at $x=0.05$. Fig.4 indicates the distribution of stress along the bar at three different nondimensional times $t=0.2488,0.5274$ and 0.7015 . As a matter of fact, authors have also worked out many comparison results for the different type of boundary conditions and material constants, but they are not shown in the present paper for the space saving. However, we may see from these comparisons that a good agreement has been arrived at between the first order approximation solution and the finite difference numerical solution even the perturbation parameter $\delta$ takes the value of 0.2 .

\section{V.Conclusions}

Some conclusions can be drown from this work :

a) The regular perturbation technique as suggested and illustrated in some detail is believed to provide a useful means to deal with the stress wave propagation in viscoelastic materials with damage.

b) The constitutive relations and damage evolution rule for visco-elastic material play a very important role in featuring the wave attenuation, the developed method including the perturbation expansion and finite difference procedure can also used as a test for the correctness of constitutive relations and damage evolution rule taken if some experimental data obtained in SHPB test are available. This experimental work will be the next goal of our study.

c) The viscoelasticity of matrix with fading memory will not produce any long terin effect on local stress distribution, however, for the damaged visco-elastic materials, the damage will be left permenant inside material and can cause a distribution of nonvanishing residual stress and strain. 


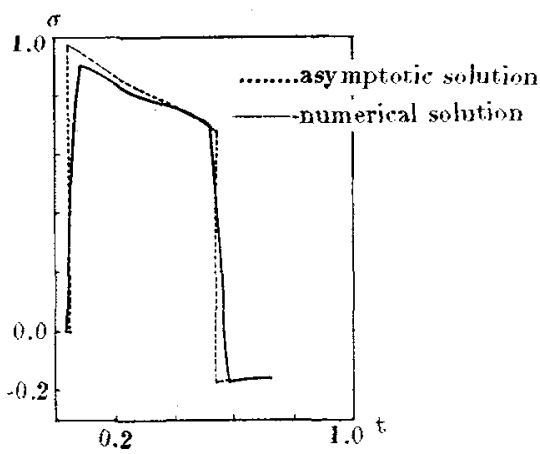

Fig.1

$$
\sigma-t \text { curve at } x=0.05
$$

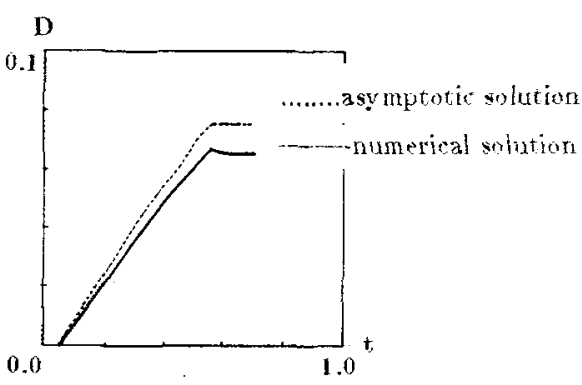

Fig.3

D-t curve at $x=0.05$

\section{Acknowledgement}

This work was supported by China Natural Science Fundation under contract No. 9187004, this support is gratefully acknowledged.

\section{References}

/1/ J.M.Chaboche, Continuum Damage Mechanics: Part I-General concepts,J.A.M.,55(1989),59 /2/J.M.Chaboche, Continuum Damage Mechanics:Part II-Damage Growth. Crack Initiation /3/L.M.Kachanov, Time of Rupture Process under Creep Conditions:Izv. Ak, Nank, sssk ctd Tekh Naul. No.8,p26

/4/Y. Weitsman, A Continum Damage Mode for viscoelastic Materials, J.A.M. 55(1985)773

/5/Lu Meng, A theory of mechanics and physics for constitutive relationships, Ph.D.dissertation, Beijing Univ. of Aero .Astro, 1989

/6/L. Davis, et al, Theory of Spalling Damage Accumulation in Ductile Materials, J. Mech. Phys. Solids, 45(1977),11

/7/R.M.Christensen, Theory of Viscoelasticity, Academic Press, INC. NewYork ,1982 